\section{RSP}

http://www.rsp.fsp.usp.br/
Revista de Saúde Pública

\title{
Intervenções educativas em serviços de saúde e saúde bucal: revisão sistemática
}

\author{
Aryane Marques Menegaz', Alexandre Emídio Ribeiro Silva,,II, Andreia Morales Cascaes ${ }^{1, \text { II }}$ \\ ' Universidade Federal de Pelotas. Faculdade de Odontologia. Programa de Pós-Graduação em Odontologia. Pelotas, \\ RS, Brasil \\ " Universidade Federal de Pelotas. Faculdade de Odontologia. Departamento de Odontologia Social e Preventiva. Pelotas, \\ RS, Brasil
}

\section{RESUMO}

OBJETIVO: Analisar a efetividade de intervenções educativas realizadas em serviços de saúde na melhoria de comportamentos e desfechos clínicos em saúde bucal.

MÉTODOS: Foi realizada uma revisão sistemática da literatura com busca nas bases de dados PubMed, Lilacs e SciELO. Foram incluídos estudos que investigaram intervenções realizadas por profissionais de saúde atuantes em serviços de saúde e que utilizaram como principal abordagem ações de educação com a finalidade de melhorar desfechos comportamentais e clínicos em saúde bucal.

RESULTADOS: A busca totalizou 832 artigos e 14 preencheram todos os critérios de inclusão. Cinco estudos verificaram exclusivamente a efetividade das intervenções na redução de cárie, três avaliaram excepcionalmente comportamentos em saúde bucal, os demais artigos julgaram a efetividade das intervenções tanto para desfechos clínicos (cárie dentária e condições periodontais) quanto para comportamentos em saúde bucal. A maioria dos estudos $(n=9)$ foi baseada em ensaios randomizados e controlados, os demais avaliaram o antes e depois da intervenção. Cinco estudos reportaram redução significativa de cárie dentária, e cinco dos seis estudos que avaliaram desfechos comportamentais encontraram alguma mudança positiva.

CONCLUSÕES: A maioria dos estudos que avaliaram desfechos comportamentais e periodontais mostrou melhorias significativas a favor das intervenções. Todos os estudos que avaliaram cárie mostraram a diminuição de novas lesões ou casos da doença nos grupos que receberam as intervenções, embora apenas cinco dos 11 artigos tenham encontrado uma diferença estatisticamente significativa. As intervenções educativas realizadas por profissionais de saúde no contexto de sua prática apresentam potencial em promover a saúde bucal da população.

DESCRITORES: Educação em Saúde Bucal. Avaliação de Eficácia-Efetividade de Intervenções. Serviços de Saúde Bucal. Revisão. 


\section{INTRODUÇÃO}

As doenças bucais são consideradas um importante problema de saúde pública não somente por sua alta prevalência ${ }^{22-24}$, mas também por afetarem negativamente a qualidade de vida dos indivíduos e demandarem altos custos para seu tratamento ${ }^{31}$. Dados do estudo mundial "Global Burden of Disease Study 2010" revelaram que cerca de 3,9 bilhões de pessoas são afetadas por doenças bucais ${ }^{34}$. A cárie não tratada em dentes permanentes foi a condição de saúde com a maior prevalência global (35\% para todas as idades combinadas) ${ }^{34}$.

O fato de as doenças bucais serem amplamente preveníveis e ainda assim apresentarem elevada prevalência é preocupante. Comportamentos saudáveis, como escovação dentária diária, contato regular com fontes de fluoretos ${ }^{35}$ e controlado consumo de açúcar ${ }^{49}$ são a forma mais efetiva de prevenir as principais doenças bucais, e também de reduzir custos aos serviços de saúde e sociedade ${ }^{47}$. O forte caráter sociocomportamental dessas doenças expõe a importância da implementação de intervenções educativas visando à apropriação do autoconhecimento sobre o processo saúde-doença, com estímulo à autonomia e mudança de comportamentos em saúde que levam a sua prevenção¹.

Revisões sistemáticas e narrativas de estudos que avaliam o efeito de intervenções educativas na melhoria da saúde bucal, publicados entre 1982 e 2013, foram previamente conduzidas. Uma revisão narrativa buscou identificar evidências de estratégias para a promoção da saúde bucal, incluindo em sua avaliação três revisões sistemáticas e duas narrativas sobre efetividade de intervenções educativas na melhoria da saúde bucal, a partir de estudos publicados entre 1982 e $1996^{59}$. As cinco revisões analisadas apresentam resultados similares quanto aos efeitos positivos a curto prazo em conhecimentos, porém, limitados em relação aos comportamentos e condições clínicas ${ }^{59}$. Cabe ressaltar que o delineamento, a metodologia e a avaliação dos estudos são criticados como sendo de baixa qualidade ${ }^{59}$.

Twetman ${ }^{55}$ revisou sistematicamente 22 estudos de diversos tipos de intervenção para prevenção de cárie em crianças até três anos de idade, publicados entre 1998 e 2007. As intervenções educativas foram relatadas em cinco estudos. $O$ autor encontrou que dois estudos de baixa qualidade apresentaram efeitos nulos, enquanto três estudos de qualidade média identificaram menor incidência de cárie no grupo que recebeu a educação em saúde em comparação ao que não recebeu. $\mathrm{O}$ autor discute que os estudos com maior efetividade foram aqueles realizados no contexto dos serviços de saúde ou que usaram a entrevista motivacional como abordagem educativa.

Lemkuhl et al. ${ }^{30}$ realizaram uma revisão narrativa de literatura que analisou o impacto de 37 estudos de intervenções educativas para a melhoria de agravos clínicos em saúde bucal, publicados entre 2003 e 2013. Efeitos inconsistentes foram encontrados para a redução de placa bacteriana, sangramento gengival, incremento de cárie e de cálculo dentário. Contudo, estudos com maior número de contatos com o público-alvo foram os que demonstraram as maiores magnitudes de efeito positivo nos desfechos investigados. A qualidade metodológica de estudos publicados recentemente ainda é fraca, segundo a análise dos autores. Ademais, a maioria das abordagens educativas utilizadas são essencialmente pautadas em modelo pedagógico individual e tradicional. Essas abordagens são criticadas por desconsideram as relações sociais, interpessoais e de contexto dos serviços de saúde.

Estudos que avaliam o efeito de intervenções educativas integradas aos serviços de saúde e que visam à melhoria da saúde bucal não foram alvo de uma revisão de caráter sistemático. Nenhuma das revisões anteriormente apresentadas classificou as intervenções integradas aos serviços de saúde como uma categoria importante de análise. Apenas Twetman ${ }^{55}$ observou que estudos desta natureza apresentam maior potencial para a prevenção de cárie em crianças. Na perspectiva das intervenções integradas aos serviços de saúde, diferentes profissionais de saúde promovem ações que aumentam a capacidade de obtenção de efeitos positivos na saúde dos indivíduos e comunidades ${ }^{62}$. A educação em saúde é listada como um dos elementos centrais no sucesso desse tipo de intervenção em diversas áreas da saúde ${ }^{14}$. 
Quanto à saúde bucal, o contato com os indivíduos e comunidades deveria ser visto por qualquer profissional de saúde como uma oportunidade para o trabalho educativo, visando a contribuir com a melhoria das condições de saúde bucal da população, especialmente em locais com acesso restrito ao dentista.

O conhecimento da efetividade das intervenções educativas para a saúde bucal auxilia na identificação das melhores estratégias a serem aplicadas no contexto das práticas dos serviços de saúde. Dada a natureza da intervenção - educativa -, a validade científica da maioria dos ensaios clínicos analisados em revisões anteriores é questionável. Diferentemente das intervenções clínicas clássicas, conduzidas em condições ótimas para produzir o efeito esperado, o sucesso nas intervenções educativas depende de uma série de atores envolvidos na sua implementação e avaliação. Nestas, as mudanças desejadas nos indivíduos devem ser acompanhadas por mudanças nas práticas profissionais e organizacionais dos serviços de saúde. Tais serviços deveriam estar voltados para uma perspectiva integrada e de apoio continuado aos usuários, utilizando a educação em saúde como ferramenta para atingir esse propósito. Entender como esse tipo de intervenção deve funcionar, no mundo real, é crucial para alcançar melhorias na saúde bucal. Diante desse contexto, a presente revisão sistemática foi conduzida com o objetivo de responder à pergunta: As intervenções educativas integradas aos serviços de saúde são efetivas em modificar comportamentos relacionados à saúde bucal ou prevenir doenças bucais?

\section{MÉTODOS}

As diretrizes do protocolo PRISMA foram seguidas para o relato desta revisão ${ }^{41}$. A revisão está registrada na plataforma PROSPERO (CRD42016052112).

A revisão sistemática de literatura foi realizada nas bases de dados PubMed, Lilacs e SciELO em janeiro de 2017. Não houve limites de datas ou língua. A literatura cinza e as referências dos artigos selecionados foram checadas para encontrar estudos adicionais eventualmente não identificados nas buscas.

A expressão utilizada na busca combinou os seguintes termos presentes no Medical Subject Headings (MeSH) e seus correspondentes no Descritores em Ciências da Saúde(DeCS):((("Health Promotion"[Mesh] OR "Health Education, Dental"[Mesh]) OR "HealthEducation"[Mesh] OR "Community Health Workers/education"[Mesh] OR "Health Personnel/education"[Mesh] OR "Education, Medical, Continuing"[Mesh] OR "Pediatrics/education"[Mesh] OR "Preventive Dentistry"[Mesh])) AND "Oral Health"[All Fields] AND ("Clinical Trial"[Publication Type] OR "Intervention Studies"[All Fields] OR "Evaluation Studies"[Publication Type] OR "Program Evaluation"[Mesh]). Um banco de dados com os resultados da pesquisa foi gerado usando a ferramenta EndNote X7.

Foram incluídos nesta revisão: (i) estudos de intervenção, com ou sem randomização, com ou sem grupo controle, abrangendo uma população de qualquer faixa etária; (ii) intervenções educativas, abrangendo saúde geral ou bucal, tendo sido executadas exclusivamente por profissionais de saúde, que exercessem sua função em serviços de saúde públicos ou privados de qualquer contexto (ex.: hospitais, centros de atenção primária à saúde, serviços de referência em universidades, clínicas médicas e odontológicas),(iii) estudos que avaliaram como desfechos melhorias em comportamentos relacionados com a saúde bucal (os principais desfechos encontrados foram: escovação diária pelo menos duas vezes ao dia, uso de mamadeira, consumo de doces, uso de serviços odontológicos) ou que avaliaram resultados em condições clínicas de saúde bucal (cárie e condições periodontais foram as únicas encontradas).

Foram excluídos estudos: (i) em que o tratamento preventivo ou curativo era a principal intervenção, visto que o enfoque da revisão consistiu em avaliar o efeito das intervenções educativas; (ii) cujo objetivo foi avaliar aquisição de conhecimentos e efeitos na prática dos estudantes ou profissionais, após treinamento para exercer ações de educação em saúde e não seu efeito na população-alvo; (iii) em escolas, com exceção daquelas em que estava explícito que tenham sido implementadas por profissionais da saúde e; (iv) com amostras muito pequenas (menor ou igual a 20 participantes). 
A seleção dos artigos iniciou por uma leitura dos títulos e resumos, realizada de forma independente por dois dos autores (AMM e AMC). A decisão final baseou-se em um terceiro revisor nos casos de desacordo (AERS). Após a seleção, os seguintes dados referentes às características dos estudos foram extraídos por dois autores (AMM e AMC): autor, amostra, local de realização do estudo (países desenvolvidos ou em desenvolvimento), tipo de estudo (ensaio randomizado e controlado ou antes e depois), tipo de serviço de saúde (centro de saúde de atenção primária, hospitais, clínica médica ou odontológica), público-alvo, tipo de recurso educativo ou estratégia utilizada, profissional que implementou a intervenção (dentista, médico, enfermeiro, agente comunitário de saúde, mais de um tipo de profissional), dose da intervenção ( 1 a 3, 4 a 12 contatos com o público-alvo), tempo máximo de avaliação em meses ( 2 a 6, 7 a 12, 13 a 24, 36 a 60), taxa de acompanhamento superior a 80\% (não ou sim) e tipo de desfecho (clínico, comportamental ou clínico e comportamental), além dos resultados obtidos nos estudos.

Os resultados sobre os desfechos foram apresentados tal qual nos artigos e, posteriormente, uma estimativa do impacto relativo das intervenções sobre os desfechos foi calculada, conforme abordagem previamente apresentada na literatura ${ }^{30}$. Foram realizados os seguintes cálculos em cada grupo: resultado final da avaliação do desfecho (RF) subtraído do resultado inicial (RI), dividido pelo resultado inicial e multiplicado por cem: $((\mathrm{RF}-\mathrm{RI}) / \mathrm{RI}) \times 100$. Quando o estudo apresentava grupo controle, os valores percentuais do grupo intervenção foram comparados ao grupo controle, por meio de cálculo análogo ao acima mencionado. Dessa forma, estimou-se, em termos percentuais, a magnitude das reduções ou aumentos dos desfechos do grupo intervenção em relação ao controle.

Foi realizada uma avaliação da qualidade dos artigos por dois dos autores (AMM e AMC). Os critérios de avaliação de qualidade foram inicialmente discutidos entre ambos e a avaliação foi feita em conjunto. As divergências foram debatidas e, por meio de consenso, tomava-se a decisão final. A qualidade dos estudos foi avaliada com o instrumento proposto por Downs e Black ${ }^{15}$, originalmente composto por 27 questões relativas à qualidade das informações do artigo, validade externa, validade interna (viés e confundimento) e poder estatístico, dando origem a uma pontuação que varia de zero a 28. A pergunta sobre a tentativa de cegar sujeitos à exposição foi excluída, uma vez que não se aplica ao tipo de intervenção realizada. Portanto, o escore dos artigos poderia variar de zero a 27. Cada estudo foi classificado, de acordo com a qualidade da evidência, como excelente (24 a 27), bom (20 a 23), razoável (15 a 19), ou pobre ou limitado (14 ou menos), conforme critérios utilizados em outra revisão ${ }^{7}$. A qualidade da evidência não foi fator de exclusão, visto que consideramos importante avaliar todas as evidências disponíveis acerca do tema e relacioná-las com os resultados encontrados.

Todos os dados foram tabulados em planilhas do programa Excel $^{\circledR}$ 2013.. As frequências absolutas e relativas foram calculadas e as estimativas do impacto relativo das intervenções foram realizadas.

\section{RESULTADOS}

As buscas resultaram em um total de 832 artigos, e após a remoção de duplicatas, restaram 830 artigos. Nesta primeira etapa foram excluídos 800 após a leitura do título e resumo, restando 30 artigos para leitura do texto na íntegra. Na sequência, um estudo foi excluído por apresentar amostra pequena $(n=20)^{3}$, nove estudos não haviam sido implementados por profissionais de saúde que atuavam no serviço ${ }^{12,13,17,21,27,28,33,45,51}$, em quatro o tratamento preventivo e curativo era a principal intervenção ${ }^{16,24,38,44}$, um estudo descrevia o protocolo e não avaliou efeitos ${ }^{53}$, e um estudo repetido foi excluído ${ }^{11}$. Ao final, quatorze estudos contemplaram todos os critérios (Figura 1) e foram incluídos na síntese qualitativa ${ }^{2,8-10,19,22,29,39,42,46,54,57,58,61}$.

A Tabela 1 resume as principais características dos estudos incluídos na revisão. Metade dos estudos foi conduzida em países em desenvolvimento ${ }^{8,19,22,39,42,46,57}$ e a grande maioria $(\mathrm{n}=9)$ incluiu como população-alvo pais e suas crianças ${ }^{2,10,22,39,46,54,57,58,61}$, com variação 


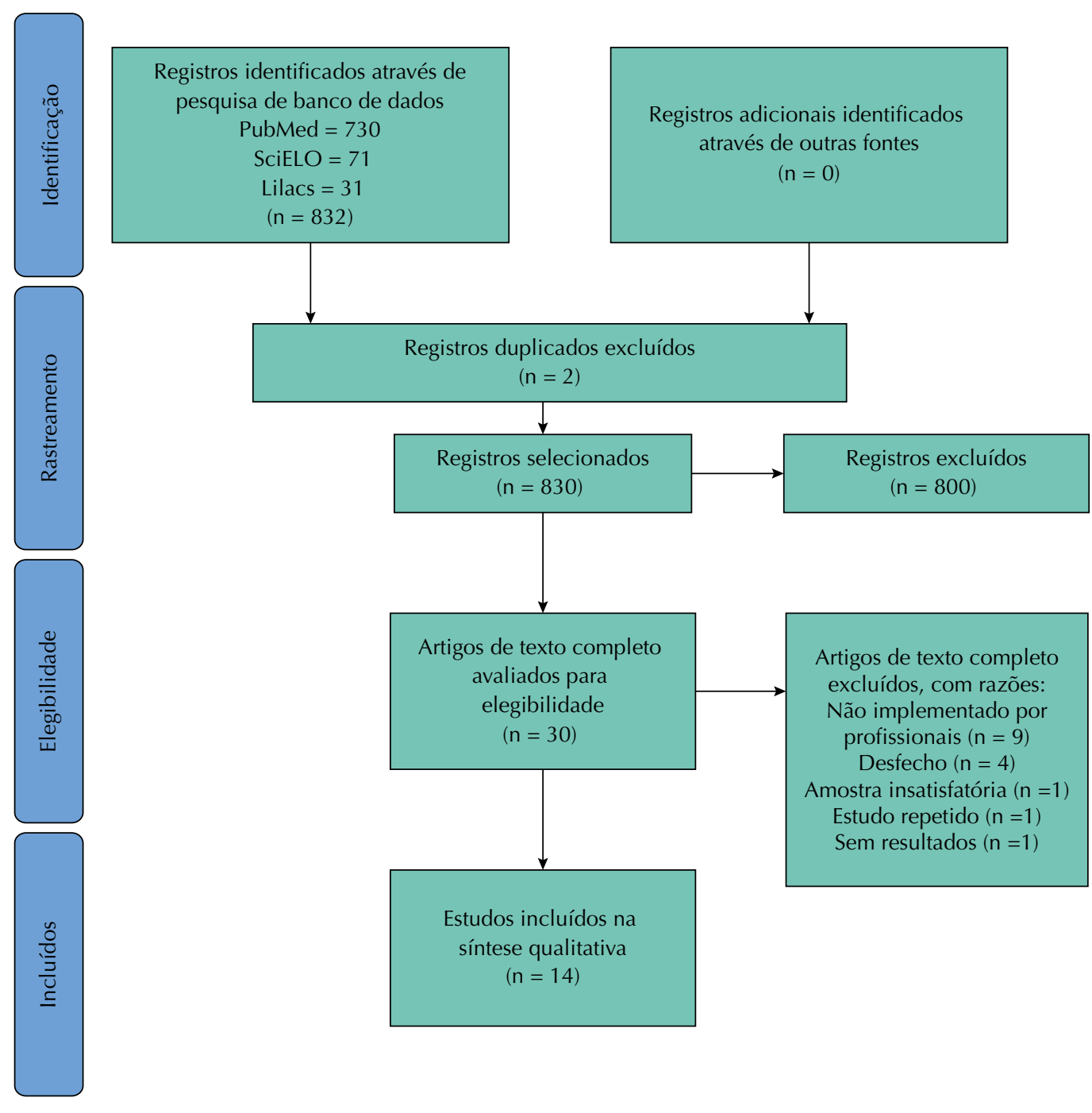

Figura. Fluxograma da seleção dos estudos. Adaptado do PRISMA statement ${ }^{41}$.

de idade de zero a oito anos. A maioria dos estudos $(n=8)$ foi baseada em ensaios clínicos randomizados controlados ${ }^{2,8-10,39,46,57,61}$ e sete foram conduzidos em centros de saúde de atenção primária ${ }^{8,10,19,39,46,57,61}$. Apenas um estudo não ofereceu aconselhamento/ orientações verbais como estratégia educativa9 ${ }^{9}$ Um estudo ofereceu apenas orientações de alimentação saudável'; os demais ofereceram, principalmente, orientações de higiene bucal. As dosagens da intervenção (i.e., número de vezes que a intervenção foi realizada em determinada população no período de tempo proposto) variaram de um a 12 contatos com o público-alvo, e metade dos estudos conduziu até três contatos ${ }^{9,19,29,39,57,58,61}$. Apenas cinco estudos obtiveram uma taxa de acompanhamento superior a $80 \%^{2,9,22,46,54}$. O tempo de acompanhamento das intervenções teve grande variação, sendo mais frequente entre sete e 12 meses $^{9,19,22,46,57}$. Cinco estudos verificaram exclusivamente a efetividade da intervenção na redução de cárie ${ }^{8,29,39,42,61}$, três avaliaram apenas comportamentos em saúde bucal ${ }^{9,19,54}$ e os demais julgaram a efetividade das intervenções tanto para desfechos clínicos (cárie dentária, placa dental, doenças periodontais) quanto para comportamentos ${ }^{2,10,22,46,57,58}$.

A avaliação da qualidade dos estudos está expressa na Tabela 2. A média do escore total foi de 17,6 pontos ( $\mathrm{DP}=4,2)$ de acordo com o instrumento de Downs e Black ${ }^{15}$. A pontuação mínima foi 11 pontos $^{42}$, e apenas dois estudos obtiveram pontuação de 25 pontos $^{8,39}$, sendo considerados excelentes. Duas intervenções foram consideradas boas ${ }^{10,57}$ e sete foram consideradas com qualidade razoável ${ }^{2,9,19,29,46,54,58}$. A avaliação segundo itens do instrumento identificou maiores problemas metodológicos relacionados a confundimento, validade externa e relato. 
Tabela 1. Descrição dos estudos incluídos na revisão. $(\mathrm{n}=14)$

\begin{tabular}{|c|c|c|}
\hline Características dos estudos & $n$ & $\%$ \\
\hline \multicolumn{3}{|l|}{ Local de realização } \\
\hline Países desenvolvidos & 7 & 50,0 \\
\hline Países em desenvolvimento & 7 & 50,0 \\
\hline \multicolumn{3}{|l|}{ Tipo de estudo } \\
\hline Ensaio randomizado e controlado & 9 & 64,2 \\
\hline Antes e depois & 5 & 35,8 \\
\hline \multicolumn{3}{|l|}{ Tipo de serviço de saúde } \\
\hline Centro de saúde de atenção primária & 7 & 50,0 \\
\hline Clínica odontológica & 3 & 21,4 \\
\hline Hospital & 3 & 21,4 \\
\hline Clínica médica & 1 & 7,2 \\
\hline \multicolumn{3}{|l|}{ Público-alvo } \\
\hline Pais e suas crianças & 9 & 64,2 \\
\hline Gestantes & 3 & 21,4 \\
\hline Crianças & 1 & 7,2 \\
\hline Mulheres usuárias de serviços públicos & 1 & 7,2 \\
\hline \multicolumn{3}{|l|}{ Tipo de recurso educativo ou estratégia utilizada* } \\
\hline Orientação/Aconselhamento verbal & 13 & 92,9 \\
\hline Entrega de panfleto/Folheto & 7 & 50,0 \\
\hline Entrega de escova e creme dental & 6 & 42,8 \\
\hline Postagem de cartazes nos serviços de saúde & 2 & 14,3 \\
\hline Demonstração de escovação em macromodelos & 2 & 14,3 \\
\hline Postagem de cartões postais via correio & 1 & 7,2 \\
\hline Demonstração em vídeos & 1 & 7,2 \\
\hline Demonstração em álbum de fotos & 1 & 7,2 \\
\hline Entrega de copo transitório & 1 & 7,2 \\
\hline Ligações & 1 & 7,2 \\
\hline \multicolumn{3}{|l|}{ Profissional que implementou a intervenção } \\
\hline Mais de um profissional & 6 & 42,8 \\
\hline Dentista & 3 & 21,4 \\
\hline Agente comunitário de saúde & 3 & 21,4 \\
\hline Médico & 1 & 7,2 \\
\hline Enfermeiro & 1 & 7,2 \\
\hline \multicolumn{3}{|l|}{ Dose da intervenção (número de contatos) } \\
\hline 1 a 3 & 7 & 50,0 \\
\hline 4 a 12 & 5 & 35,8 \\
\hline Não informado & 2 & 14,2 \\
\hline \multicolumn{3}{|l|}{ Tempo máximo de avaliação (meses) } \\
\hline 2 a 6 & 3 & 21,4 \\
\hline 7 a 12 & 5 & 35,8 \\
\hline 13 a 24 & 1 & 7,2 \\
\hline 36 a 60 & 3 & 21,4 \\
\hline Não informado & 2 & 14,2 \\
\hline \multicolumn{3}{|l|}{ Taxa de acompanhamento superior a $80 \%$} \\
\hline Não & 7 & 50,0 \\
\hline Sim & 5 & 35,8 \\
\hline Não informado & 2 & 14,2 \\
\hline \multicolumn{3}{|l|}{ Desfechos } \\
\hline Clínicos & 6 & 42,8 \\
\hline Comportamentais & 3 & 21,4 \\
\hline Clínicos e comportamentais & 5 & 35,8 \\
\hline
\end{tabular}

* Alguns estudos utilizaram mais de um tipo de recurso; os valores percentuais apresentados são individuais para cada tipo de recurso.

A Tabela 3 engloba os resultados das intervenções sobre os comportamentos em saúde bucal analisados. Seis estudos avaliaram escovação diária de no mínimo duas vezes ao dia; a maioria $(\mathrm{n}=5)$ apresentou resultados positivos em favor das intervenções, ${ }^{910,46,57,58}$, com magnitudes de efeito que variaram de $11,0 \%{ }^{9}$ a $141,5 \%{ }^{46}$. Diferenças significativas também foram encontradas quanto ao uso de mamadeira e consumo de doces para a maioria dos estudos; cinco dos seis estudos demonstraram melhoria nesses desfechos nos grupos que receberam as intervenções ${ }^{9,10,22,54,57}$, cujas magnitudes de efeito variaram de $7,6 \%{ }^{54}$ a 83,3\% ${ }^{10}$. Por fim, os três estudos que se propuseram a avaliar aumento no uso do serviço odontológico encontraram efeitos positivos da intervenção ${ }^{9,19,58}$.Em dois deles ${ }^{9,19}$, observou-se as maiores magnitudes de efeito em relação a todos os comportamentos avaliados. 
Tabela 2. Avaliação da qualidade das intervenções, de acordo com os critérios de Downs e Black ${ }^{15}$.

\begin{tabular}{|c|c|c|c|c|c|c|}
\hline Autores, ano & $\frac{\text { Relato }}{\text { (0 a 10) }}$ & $\frac{\text { Validade externa }}{(0 \text { a } 3)}$ & $\begin{array}{l}\text { Viés* } \\
\text { (0 a 6) }\end{array}$ & $\frac{\text { Confundimento }}{(0 \mathrm{a} 6)}$ & $\begin{array}{l}\text { Poder } \\
\text { (0 a } 1) \\
\end{array}$ & $\begin{array}{c}\frac{\text { Somatório }}{\text { (0 a 26) }} \\
\end{array}$ \\
\hline Chaffee et al. ${ }^{8}(2013)$ & 10 & 3 & 5 & 6 & 1 & 25 \\
\hline Mohebbi et al. ${ }^{39}$ (2007) & 9 & 3 & 6 & 6 & 1 & 25 \\
\hline Vachirarojpsian et al. ${ }^{57}$ (2005) & 8 & 3 & 6 & 5 & 0 & 22 \\
\hline Davies et al. ${ }^{10}(2005)$ & 8 & 3 & 5 & 3 & 1 & 20 \\
\hline Raj et al. ${ }^{46}(2003)$ & 8 & 3 & 5 & 2 & 1 & 19 \\
\hline Blinkhorn et al. ${ }^{2}$ (2003) & 6 & 1 & 6 & 3 & 1 & 17 \\
\hline Cibuka et al. ${ }^{9}(2011)$ & 9 & 0 & 5 & 2 & 1 & 17 \\
\hline Frazão et al. ${ }^{19}$ (2009) & 7 & 3 & 4 & 2 & 1 & 17 \\
\hline Wagner et al..$^{58}(2013)$ & 7 & 3 & 3 & 3 & 1 & 17 \\
\hline Strippel et al. ${ }^{54}(2010)$ & 8 & 0 & 4 & 3 & 1 & 16 \\
\hline Larsen et al. ${ }^{29}(2016)$ & 8 & 1 & 4 & 1 & 1 & 15 \\
\hline Gauba et al. ${ }^{22}(2016)$ & 8 & 0 & 4 & 1 & 1 & 13 \\
\hline Whittle et al. ${ }^{61}$ (2008) & 5 & 0 & 5 & 2 & 1 & 13 \\
\hline Moskovitz et al. ${ }^{42}$ (2009) & 7 & 0 & 2 & 2 & 0 & 11 \\
\hline Média (DP) & $7,7(1,3)$ & $1,6(1,4)$ & $4,6(1,2)$ & $2,9(1,6)$ & $0,9(0,4)$ & $17,6(4,2)$ \\
\hline
\end{tabular}

* Questão 14 do instrumento Downs e Black foi excluída.

Tabela 3. Resultados das intervenções sobre os principais comportamentos em saúde bucal.

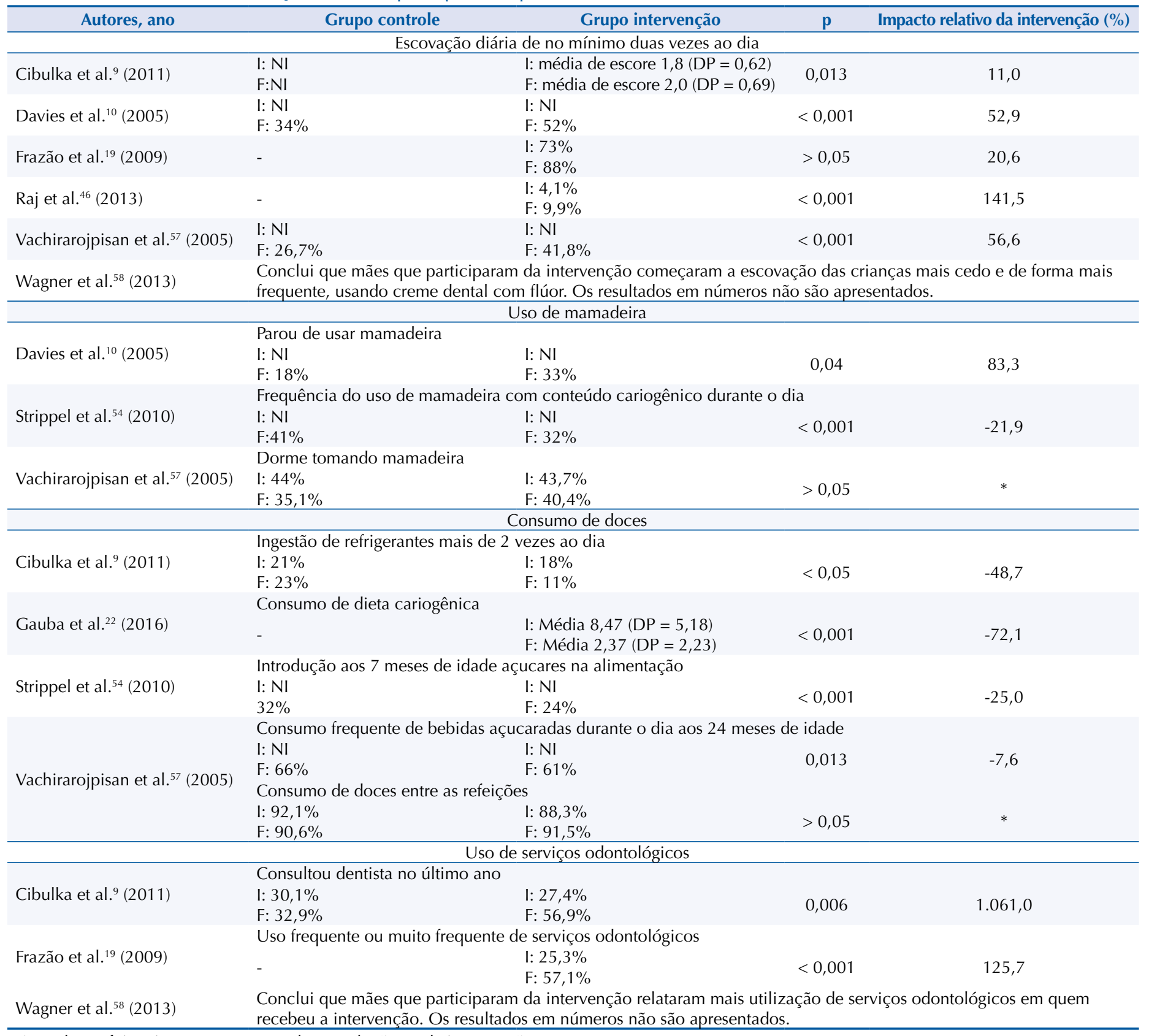

* A mudança foi maior no grupo controle em relação ao de intervenção. 
Os resultados quanto à redução de doenças e agravos bucais estão expressos na Tabela 4. Dos 11 estudos que avaliaram prevenção de novas lesões/casos de cárie, cinco apresentaram diferenças significativas ao fim de suas intervenções ${ }^{10,22,29,42,58}$, com magnitudes de efeito variando de $31,6 \%{ }^{10}$ a $481,6 \%^{22}$. Embora a maioria das intervenções $(n=6)$ não tenha apresentado diferenças significativas para a doença cárie, todos os estudos demonstraram uma diminuição de cárie dentária nos grupos que receberam as intervenções. Ainda, outros dois estudos ${ }^{2,46}$ trataram do desfecho clínico placa dental e um para cálculo ${ }^{46}$, observando-se resultados positivos.

Tabela 4. Resultados das intervenções para os desfechos clínicos.

\begin{tabular}{|c|c|c|c|c|c|}
\hline Autores, ano & Índice & Grupo controle & Grupo intervenção & $\mathbf{p}$ & $\begin{array}{l}\text { Impacto relativo da } \\
\text { intervenção }(\%)\end{array}$ \\
\hline \multicolumn{6}{|c|}{ Cárie dentária } \\
\hline Blinkhorn et al. ${ }^{2}$ (2003) & ceo-d & $\begin{array}{l}\text { I: } 2,2(\text { DP } 2,3) \\
\text { F: } 3,2(\text { DP } 2,8)\end{array}$ & $\begin{array}{l}\text { I: } 2,0(\text { DP } 2,2) \\
\text { F: } 2,6(\text { DP } 2,6)\end{array}$ & 0,21 & $-33,3$ \\
\hline \multirow{2}{*}{ Chaffee et al. ${ }^{8}$ (2013) } & ceo-s médio (com manchas brancas) & $\begin{array}{l}\text { I: zero } \\
\text { F: } 3,6(\text { DP } 6,9)\end{array}$ & $\begin{array}{l}\text { I: zero } \\
\text { F: } 2,8(\text { DP } 5,4)\end{array}$ & 0,25 & $-22,2$ \\
\hline & $\begin{array}{l}\text { média do componente cariado do } \\
\text { ceo-s }\end{array}$ & $\begin{array}{l}\text { I: zero } \\
\text { F: } 3,0(\text { DP } 6,8)\end{array}$ & $\begin{array}{l}\text { I: zero } \\
\text { F: } 2,1(\text { DP } 5,0)\end{array}$ & 0,18 & $-30,0$ \\
\hline \multirow{2}{*}{ Davies et al. ${ }^{10}(2005)$} & ceo-d médio & $\begin{array}{l}\text { I: NI } \\
\text { F: } 1,7\end{array}$ & $\begin{array}{l}\text { I: NI } \\
\text { F: } 1,1\end{array}$ & $<0,001$ & $-35,3$ \\
\hline & ceo-s médio & $\begin{array}{l}\text { I: NI } \\
\text { F: } 3,8\end{array}$ & $\begin{array}{l}\text { I: NI } \\
\text { F: } 2,6\end{array}$ & 0,008 & $-31,6$ \\
\hline Gauba et al. ${ }^{22}(2016)$ & $\begin{array}{l}\text { Não utilizou índice. Baseou-se em } \\
\text { critério próprio e caracterizou-o como } \\
\text { "chance de evitar novas cavidades" }\end{array}$ & - & $\begin{array}{l}\text { I: Média 12,5 (DP 13,5) } \\
\text { F: Média 72,7 (DP 14,4) }\end{array}$ & $<0,001$ & 481,6 \\
\hline Larsen et al. ${ }^{29}(2016)$ & $\begin{array}{c}\text { Não utilizou índice. Presença ou } \\
\text { ausência de cárie }\end{array}$ & $\begin{array}{l}\text { I: zero } \\
\text { F: } 29 \text { crianças com } \\
\text { ausência de cárie }(69 \%)\end{array}$ & $\begin{array}{l}\text { I: zero } \\
\text { F: } 19 \text { crianças com } \\
\text { ausência de cárie }(39 \%)\end{array}$ & 0,015 & $-43,5$ \\
\hline \multirow{2}{*}{ Mohebbi et al. ${ }^{39}(2007)$} & $\begin{array}{l}\text { Média de cárie em esmalte } \\
\text { Critério OMS (1997) }\end{array}$ & $\begin{array}{l}\text { I: } 0,08 \text { (DP } 0,4) \\
\text { F: } 0,48(\text { DP } 1,0)\end{array}$ & $\begin{array}{l}\text { Grupo A } \\
\text { I: 0,25 (DP 0,7) } \\
\text { F: 0,15 (DP 0,5) } \\
\text { Grupo B } \\
\text { I: } 0,25 \text { (DP } 0,7) \\
\text { F: 0,35 (DP } 1,0)\end{array}$ & 0,283 & $\begin{array}{c}\text { Grupo A } \\
-108,0 \\
\text { Grupo B } \\
-92,0\end{array}$ \\
\hline & $\begin{array}{l}\text { Média de cárie em dentina } \\
\text { Critério OMS (1997) }\end{array}$ & $\begin{array}{l}\text { I: } 0,03(\text { DP } 0,25) \\
\text { F: } 0,23(\text { DP } 0,95)\end{array}$ & $\begin{array}{l}\text { Grupo A } \\
\text { I: 0,04 (DP 0,2) } \\
\text { F: 0,02 (DP 0,2) } \\
\text { Grupo B } \\
\text { I: } 0,14 \text { (DP 0,8) } \\
\text { F: 0,12 (DP 0,3) }\end{array}$ & 0,719 & $\begin{array}{l}\text { Grupo A } \\
-107,6 \\
\text { Grupo B } \\
-102,1\end{array}$ \\
\hline \multirow{2}{*}{ Moskovitz, et al. ${ }^{42}(2009)$} & $\begin{array}{l}\text { Média componente cariado do } \\
\text { CPO-D }\end{array}$ & $\begin{array}{l}\text { I: NI } \\
\text { F: } 5,9\end{array}$ & $\begin{array}{l}\text { I: NI } \\
\text { F: } 3,2\end{array}$ & $<0,001$ & $-45,8$ \\
\hline & CPO-D médio & $\begin{array}{l}\text { I: NI } \\
\text { F: } 7,5\end{array}$ & $\begin{array}{l}\text { I: NI } \\
\text { F: } 6,8\end{array}$ & $>0,05$ & $-9,3$ \\
\hline Raj et al. ${ }^{46}(2013)$ & ceo-d médio & - & $\begin{array}{l}\text { I: } 2,1(\text { DP } 3,2) \\
\text { F: } 1,9(\text { DP } 1,4)\end{array}$ & 0,060 & $-9,5$ \\
\hline Vachirarojpisan et al. ${ }^{57}$ (2005) & $\begin{array}{l}\text { Lesões de cárie cavitadas } \\
\text { e não-cavitadas* }\end{array}$ & $\begin{array}{l}\text { I: } 1,7 \text { (DP 2,6) } \\
\text { F: } 7,7 \text { (DP 5,2) }\end{array}$ & $\begin{array}{l}\text { I: } 1,9 \text { (DP 2,7) } \\
\text { F: } 7,8 \text { (DP 5,0) }\end{array}$ & $>0,05$ & $-11,4$ \\
\hline \multirow{2}{*}{ Wagner et al..$^{58}(2013)$} & ceo-s médio & $\begin{array}{l}\text { I: zero } \\
\text { F: } 5,2(\text { DP } 6,4)\end{array}$ & $\begin{array}{l}\text { I: zero } \\
\text { F: } 3,2(\mathrm{DP} 7,4)\end{array}$ & $<0,05$ & $-38,5$ \\
\hline & ceo-d médio & $\begin{array}{l}\text { I: zero } \\
\text { F: } 2,4(\text { DP } 4,1)\end{array}$ & $\begin{array}{l}\text { I: zero } \\
\text { F: } 1,5(\text { DP } 2,5)\end{array}$ & $<0,05$ & $-37,5$ \\
\hline Whittle et al. ${ }^{61}$ (2008) & ceo-s médio & $\begin{array}{l}\text { I: zero } \\
\text { F: } 2,2(36 \text { meses }) \\
\text { I: zero } \\
\text { F: } 4,8 \text { ( } 60 \text { meses })\end{array}$ & $\begin{array}{l}\text { I: zero } \\
\text { F: } 2,0 \text { (36 meses) } \\
\text { I: zero } \\
\text { F: } 4,0 \text { ( } 60 \text { meses }) \\
\end{array}$ & $>0,05$ & $-15,2$ \\
\hline \multicolumn{6}{|c|}{ Condições periodontais } \\
\hline Blinkhorn et al. $^{2}$ (2003) & Placa dental - índice NI & $\begin{array}{l}\text { I: } \mathrm{NI} \\
\mathrm{F}: 61 \%\end{array}$ & $\begin{array}{l}\text { I: } \mathrm{NI} \\
\mathrm{F}: 53 \%\end{array}$ & 0,16 & $-13,1$ \\
\hline \multirow{3}{*}{ Raj et al. ${ }^{46}(2013)$} & Placa dental - índice NI & - & $\begin{array}{l}\text { I: } 75,5 \% \\
\text { F: } 66,5 \%\end{array}$ & $<0,001$ & $-11,9$ \\
\hline & Sangramento gengival & - & $\begin{array}{l}\text { I: } 1,7 \% \\
\text { F: } 2,2 \%\end{array}$ & 0,671 & 29,4 \\
\hline & Cálculo & - & $\begin{array}{l}\text { I: } 78,3 \% \\
\text { F: } 54,1 \%\end{array}$ & $<0,001$ & $-30,9$ \\
\hline
\end{tabular}

I: exame inicial; F: exame final; NI: não informado; ceo-s: índice de superfícies de dentes decíduos cariados, extraídos e restaurados devido à cárie; ceo-d: índice de dentes decíduos cariados, extraídos e restaurados devido à cárie; CPO-D: índice de dentes permanentes cariados, extraídos e restaurados devido à cárie. 


\section{DISCUSSÃO}

Os resultados desta revisão mostram que as intervenções educativas promovem mudanças de comportamentos relacionados à saúde bucal. As magnitudes de efeito das intervenções para esses desfechos foram decrescentes na seguinte ordem: uso frequente de serviços odontológicos, higiene bucal adequada e redução do consumo de doces. A evidência de efetividade para alguns desfechos periodontais, como placa e cálculo dental, élimitada a apenas dois estudos ${ }^{2,46}$, de qualidade razoável, que identificaram melhorias significativas. Ainda que todas as intervenções educativas tenham observado redução de novas lesões/casos de cárie, apenas cinco estudos, de um total de 11, reportaram essas diferenças como significativas. Dentre esses cinco estudos, apenas um foi classificado como sendo de boa qualidade metodológica ${ }^{10}$.

A estimativa de magnitude de efeitos foi bastante variável entre desfechos de uma mesma categoria. Diferentemente de outros tipos de intervenção, em que o desfecho é estimado em termos de taxa de mortalidade ou pontos de corte bem definidos de morbidade, para as intervenções educativas não há um parâmetro ideal estabelecido de taxa de mudança ou magnitude de efeito desejado e relevante para a saúde pública. Análises combinadas entre benefícios e custos associados ou a definição de desfechos que representem o número de indivíduos que devem ser tratados para que se obtenha um resultado favorável poderiam ser incorporadas aos estudos. Abordagens como essa contribuem para reduzir a limitação existente na interpretação dos resultados desse tipo de intervenção, bem como estreitar as lacunas entre subjetividade e ciência.

A qualidade das evidências para a maioria dos estudos foi classificada como pobre/limitada ou razoável. Os principais problemas que levaram a essa classificação estão relacionados: ao tipo de delineamento (antes e depois); à descrição insuficiente da definição e validade dos desfechos, principalmente os desfechos de natureza comportamental; à falta de detalhamento na descrição das intervenções; à ausência de monitoramento e fidelidade de implementação dos protocolos; à perda alta de acompanhamento e falta de relato das características dos participantes perdidos; à falta de descrição da população que deu origem à amostra, bem como dos critérios utilizados na sua seleção; e à ausência de cálculo amostral e problemas na análise e apresentação dos resultados.

O efeito positivo em relação aos comportamentos em saúde bucal pode ser um indicativo de sucesso dessas intervenções para a prevenção de doenças, uma vez que o primeiro passo para a manutenção da saúde bucal é a construção de comportamentos saudáveis. O grande efeito observado no uso de serviços odontológicos reflete o resultado de intervenções que vincularam estratégias para facilitar o acesso ao dentista, indicando a importância que as intervenções integradas aos serviços de saúde têm sobre os indivíduos. Nesse sentido, a utilização de serviços de saúde não é apenas um comportamento individual, mas um reflexo do contexto das práticas profissionais e de organização dos serviços. Serviços que apresentam uma boa organização do acesso e que atuam sob uma perspectiva integrada de saúde aumentam sua utilização preventiva por parte da população. Indivíduos que utilizam serviços de saúde regularmente têm maior oportunidade de receber diagnósticos precoces, adquirir conhecimentos e modificar comportamentos em prol de sua saúde, reduzindo a probabilidade de desenvolver problemas de saúde ${ }^{4,56}$.

A mensuração de comportamentos de higiene e consumo de doces é realizada por meio de perguntas autorreferidas, passíveis de erros de informação. Além disso, tais comportamentos são, geralmente, avaliados por meio de perguntas que apresentam validade limitada ${ }^{6}$. $\mathrm{O}$ fato de serem autorreferidos e a natureza da intervenção ser educativa pode refletir o conhecimento adquirido e não uma mudança real de comportamento. A utilização de medidas ou instrumentos válidos para mensurar comportamentos, bem como a combinação desses com a avaliação de desfechos clínicos, contribuem com o entendimento dos efeitos das intervenções dessa natureza.

Para que se observem efeitos positivos em desfechos clínicos de saúde bucal como cárie, alguns aspectos metodológicos dos estudos devem ser levados em consideração. O uso de amostras insuficientes, o pequeno número de contatos com a população-alvo e o tempo 
de acompanhamento inferior a 12 meses podem ter levado à ausência de efeito em muitos dos estudos. Evidências indicam que o tempo de progressão para ocorrência de cárie varia de acordo com a idade, podendo ser em torno de seis a 12 meses em crianças pequenas, e 24 a 36 meses em adultos ${ }^{18,50}$. Contudo, para identificar diferenças significativas entre os grupos, é também necessário realizar adequado cálculo amostral, considerando o número e o tamanho dos aglomerados ${ }^{25}$ no caso de intervenções comunitárias. As pesquisas tenderam a apresentar cálculos amostrais sem considerar o nível de aglomeração dos participantes, limitando a validade interna dos achados ${ }^{40,48}$.

A simples comparação dos grupos por meio de testes de proporção e média não garante a significância dos resultados relatados nos estudos. Salvo exceção ${ }^{8}$, os estudos não apresentaram nenhuma avaliação das taxas de mudança e dos efeitos relativos das intervenções sobre os desfechos. Os impactos relativos das intervenções, calculados pelos autores desta revisão, são apenas estimativas, em termos percentuais, da magnitude dos efeitos. O cálculo adequado das medidas de risco relativo considera as taxas de mudança nos grupos, as quais dependem do tempo de acompanhamento e do número de indivíduos que iniciaram e concluíram o estudo. Sugere-se que futuros estudos considerem esses cálculos na avaliação dos seus resultados, apresentando estimativas mais reais dos efeitos das intervenções.

A dosagem da intervenção, ou seja, o número de contatos com a população-alvo, é outro fator importante para o sucesso na mudança de comportamentos e consequente redução de doenças. Conforme discutido previamente na literatura, intervenções educativas com no mínimo quatro contatos com o público-alvo apresentam maiores chances de sucesso ${ }^{7,30}$. As evidências encontradas na presente revisão não deixam claro qual seria o número mínimo de contatos necessários para que se observem os efeitos desejados. A relação entre dose e efeitos variou amplamente, não sendo possível detectar essas diferenças entre as intervenções.

As últimas evidências disponíveis na literatura acerca da efetividade das intervenções educativas $^{23,26}$, assim como esta revisão, sugerem a necessidade de padrões científicos mais rigorosos no monitoramento das intervenções. Nos estudos avaliados na presente revisão, observou-se que a falta de avaliação da implementação das intervenções e a ausência de modelos teóricos claros dificultam o entendimento de como as intervenções funcionam ou deveriam funcionar. Atenção especial deve ser dada à fidelidade das intervenções, que se refere à medida que uma intervenção adere ao modelo original e a sua avaliação ${ }^{52}$. Quanto maior a fidelidade ao protocolo da intervenção mais positivos são os resultados nos desfechos ${ }^{5,36}$. A fidelidade de uma intervenção pode ser medida por diferentes métodos: estratégias quantitativas e qualitativas, escalas-modelo específicas, checklists com os elementos-chave da intervenção, entrevistas com profissionais ou pacientes, grupos focais, consulta aos prontuários, análise de vídeos da intervenção e entrevistas por telefone ${ }^{5}$.

Em síntese, os resultados incluídos na presente revisão sistemática são de difícil comparação, i.e., o tipo de intervenção educativa que é mais eficaz permanece pouco claro, uma vez que os protocolos diferem grandemente entre os estudos. Os tipos de abordagem educativa apresentaram grande variabilidade; entretanto, foi detectado que intervenções entregues com mais de um método associado (sessão de vídeo, folheto impresso com orientações, aconselhamento verbal e práticas de escovação) apresentaram melhorias significativas na redução de cárie dentária ${ }^{10,42,58}$. Contudo, mais importante do que o recurso pedagógico é a qualidade das abordagens educacionais, que deve ser personalizada, de acordo com o contexto sociocultural e, preferencialmente, baseada em teorias comportamentais ${ }^{60}$.

Intervenções educativas integradas aos serviços de saúde tornam-se relevantes para o fortalecimento dos sistemas de saúde, uma vez que aumentam a chance de sustentabilidade e apoio continuado, resultando em efeitos benéficos em longo razo $^{62}$. Entretanto, para que esses resultados sejam concretizados, é preciso qualificar as abordagens educativas utilizadas pela maioria dos profissionais de saúde, as quais foram retratadas em boa parte dos estudos analisados como sendo tradicionais. Abordagens pautadas apenas na transmissão de conhecimentos têm sido alvo de severas críticas, uma vez que transferem para os indivíduos 
a responsabilidade por sua saúde, produzindo nenhum ou poucos efeitos ${ }^{60}$. As abordagens educativas precisam ser apropriadas não somente aos aspectos individuais, mas também adequadas às relações sociais e interpessoais dos sujeitos.

A Entrevista Motivacional é uma técnica de abordagem centrada e personalizada ao indivíduo, buscando auxiliá-lo na resolução de seus dilemas e no alcance da motivação necessária para a mudança de comportamentos em saúde ${ }^{37}$. Apresenta caráter colaborativo, evocativo e com respeito pela autonomia do indivíduo. Quando realizada de forma efetiva, aumenta a probabilidade de engajamento do indivíduo na mudança comportamental. É uma abordagem alternativa à tradicional, com evidências de efetividade para diversas áreas da saúde ${ }^{32,43}$. Em relação à saúde bucal, duas revisões sistemáticas incluíram, ao total, 18 estudos que investigaram os efeitos da Entrevista Motivacional na saúde bucal comparada à abordagem educativa tradicional (ex., transmissão de conhecimentos) ou à nenhuma abordagem ${ }^{7,20}$. As evidências são inconclusivas, uma vez que os estudos relatam resultados controversos, variando entre efeitos positivos e nulos para a prevenção de cárie e doença periodontal. Apesar disso, os autores interpretaram com otimismo o potencial dessa abordagem, uma vez que há efetividade comprovada em ouras áreas. Maiores cuidados metodológicos no que se refere a delineamento, monitoramento e avaliação dessas intervenções em futuros estudos são recomendados a fim de melhorar a qualidade das evidências ${ }^{7,20}$.

Os pontos fortes da presente revisão incluem a seleção e a avaliação dos artigos por pares, a inclusão de um instrumento padronizado para avaliação da qualidade metodológica de estudos de intervenção e a inexistência de outra revisão publicada com enfoque no contexto de intervenções educativas integradas aos serviços de saúde, o que reforça a relevância dos resultados para a saúde pública. Dentre as limitações desta revisão, podem ser mencionadas a não inclusão de todas as bases de dados existentes e a impossibilidade de realização de síntese estatística por meio de meta-análise, devido à heterogeneidade metodológica e população-alvo das intervenções existentes.

Pode-se concluir que as intervenções realizadas por profissionais de saúde no cotidiano de sua prática resultaram na melhoria de comportamentos relacionados com a saúde bucal na maioria dos estudos. Todos os estudos apresentaram uma redução de novas lesões/casos de cárie, mesmo que essa não tenha sido sempre significativa. A grande maioria dos estudos teve como público-alvo crianças; portanto, a evidência da efetividade dessas intervenções para outros grupos populacionais ainda é desconhecida. Para reforçar as evidências, são necessários estudos futuros com abrangência de adultos e idosos, com maior qualidade metodológica e embasamento teórico das intervenções e melhor relato nas publicações e análise dos resultados.

\section{REFERÊNCIAS}

1. Alves GG, Aerts D. As práticas educativas em saúde e a Estratégia Saúde da Família. Cien Saude Colet. 2011;16(1):319-25. https://doi.org/10.1590/S1413-81232011000100034

2. Blinkhorn AS, Gratrix D, Holloway PJ, Wainwright-Stringer YM, Ward SJ, Worthington HV. A cluster randomised, controlled trial of the value of dental health educators in general dental practice. Br Dent J. 2003;195(7):395-400. https://doi.org/10.1038/sj.bdj.4810566

3. Brown RM, Canham D, Cureton VY. An oral health education program for Latino immigrant parents. J Sch Nurs. 2005;21(5):266-71. https://doi.org/10.1177/10598405050210050401

4. Camargo MB, Dumith SC, Barros AJ. [Regular use of dental care services by adults: patterns of utilization and types of services]. Cad Saude Publica. 2009;25(9):1894-906. https://doi.org/10.1590/S0102-311X2009000900004

5. Campbell BK. Fidelity in public health clinical trials: considering provider-participant relationship factors in community treatment settings. J Public Health Dent. 2011;71(1):64-5. https://doi.org/10.1111/j.1752-7325.2011.00227.x

6. Cascaes AM, Peres KG, Peres MA, Demarco FF, Santos I, Matijasevich A, et al. Validity of 5-year-old children's oral hygiene pattern referred by mothers. Rev Saude Publica. 2011;45(4):668-75. https://doi.org/10.1590/S0034-89102011005000033 
7. Cascaes AM, Bielemann RM, Clark VL, Barros AJ. Effectiveness of motivational interviewing at improving oral health: a systematic review. Rev Saude Publica. 2014;48(1):142-53. https://doi.org/10.1590/S0034-8910.2014048004616

8. Chaffee BW, Feldens CA, Vitolo MR. Cluster-randomized trial of infant nutrition training for caries prevention. J Dent Res. 2013;92(7):29-36. https://doi.org/10.1177/0022034513484331

9. Cibulka NJ, Forney S, Goodwin K, Lazaroff P, Sarabia R. Improving oral health in low-income pregnant women with a nurse practitioner-directed oral care program. J Am Acad Nurse Pract. 2011;23(5):249-57. https://doi.org/10.1111/j.1745-7599.2011.00606.x

10. Davies GM, Duxbury JT, Boothman NJ, Davies RM, Blinkhorn AS. A staged intervention dental health promotion programme to reduce early childhood caries. Community Dent Health. $2005 ; 22(2): 118-22$.

11. Davies GM, Duxbury JT, Boothman NJ, Davies RM. Challenges associated with the evaluation of a dental health promotion programme in a deprived urban area. Community Dent Health. 2007;24(2):117-21.

12. Dermen KH, Ciancio SG, Fabiano JA. A pilot test of motivational oral health promotion with alcohol-dependent inpatients. Health Psychol. 2014;33(4):392-5. https://doi.org/10.1037/a0033153

13. Dobarganes Coca AM, Lima Álvarez M, López Larquin N, Pérez Cedrón RA, González Vale L. Intervención educativa en salud bucal para gestantes. Arch Med Camaguey. 2011;15(3):528-41. Disponível em: http://scielo.sld.cu/pdf/amc/v15n3/amc100311.pdf

14. Dorling G, Fountaine T, McKenna S, Suresh B. Healthcare practice: the evidence for integrated care. McKinsey \& Company; 2015.

15. Downs SH, Black N. The feasibility of creating a checklist for the assessment of the methodological quality both of randomised and non-randomised studies of health care interventions. J Epidemiol Community Health. 1998;52(6):377-84. https://doi.org/10.1136/jech.52.6.377

16. Ekstrand KR, Kuzmina IN, Kuzmina E, Christiansen ME. Two and a half-year outcome of cariespreventive programs offered to groups of children in the Solntsevsky district of Moscow. Caries Res. 2000;34(1):8-19. https://doi.org/10.1159/000016564

17. Farias DG, Leal SC, de Toledo OA, Faber J, Bezerro AC. Effect of oral anticipatory guidance on oral health and oral hygiene practices in preschool children. J Clin Pediatr Dent. 2005;30(1):23-7. https://doi.org/10.17796/jcpd.30.1.f09716417v202263

18. Fejerskov O, Kidd E. Cárie Dentária: A Doença e seu Tratamento Clínico. $2^{a}$ ed. São Paulo: Santos; 2013

19. Frazao P, Marques D. Effectiveness of a community health worker program on oral health promotion. Rev Saude Publica. 2009;43(3):463-71. https://doi.org/10.1590/S0034-89102009005000016

20. Gao X, Lo EC, Kot SC, Chan KC. Motivational interviewing in improving oral health: a systematic review of randomized controlled trials. J Periodontol. 2014;85(3):426-37. https://doi.org/10.1902/jop.2013.130205

21. Gathece LW, Wang'ombe JK, Ng'ang'a PM, Wanzala PN. Effect of health education on knowledge and oral hygiene practices of persons living with HIV in Nairobi, Kenya. East Afr J Public Health. 2011;8(3):207-11.

22. Gauba K, Goyal A, Mittal N. A CAMBRA Model For High Caries Risk Indian Children: A Pragmatic Comprehensive Tailored Intervention. J Clin Pediatr Dent. 2016;40(1):36-43. https://doi.org/10.17796/1053-4628-40.1.36

23. Habbu SG, Krishnappa P. Effectiveness of oral health education in children - a systematic review of current evidence (2005-2011). Int Dent J. 2015;65(2):57-64. https://doi.org/10.1111/idj.12137

24. Harrison RL, MacNab AJ, Duffy DJ, Benton DH. Brighter Smiles: Service learning, interprofessional collaboration and health promotion in a First Nations community. Can J Public Health. 2006;97(3):237-40. https://doi.org/10.17269/cjph.97.730

25. Hemming K, Taljaard M. Sample size calculations for stepped wedge and cluster randomised trials: a unified approach. J Clin Epidemiol. 2016;69:137-46. https://doi.org/10.1016/j.jclinepi.2015.08.015

26. Kay EJ, Locker D. Is dental health education effective? A systematic review of current evidence. Community Dent Oral Epidemiol. 1996;24(4):231-5. https://doi.org/10.1111/j.1600-0528.1996.tb00850.x

27. Kowash MB, Pinfield A, Smith J, Curzon ME. Effectiveness on oral health of a long-term health education programme for mothers with young children. Br Dent J. 2000;188(4):201-5. https://doi.org/10.1038/sj.bdj.4800431 
28. Kuhn E, Wambier DS. Incidência de lesões de cárie em bebês após 15 meses de um programa educativo-preventivo. Pesqui Bras Odontopediatria Clin Integr. 2007;7(1):75-81. https://doi.org/10.4034/1519.0501.2007.00711.0011

29. Larsen CD, Larsen MD, Ambrose T, Degano R, Gallo L, Cardo VA, Jr. Efficacy of a Prenatal Oral Health Program Follow-up with Mothers and their Children. N Y State Dent J. 2016;82(3):15-20.

30. Lemkuhl I, Souza MVC, Cascaes AM, Bastos JL. A efetividade das intervenções educativas em saúde bucal: revisão de literatura. Cad Saude Colet. 2015;23(3):336-46. https://doi.org/10.1590/1414-462X201400030104

31. Listl S, Galloway J, Mossey PA, Marcenes W. Global Economic Impact of Dental Diseases. J Dent Res. 2015;94(10):1355-61. https://doi.org/10.1177/0022034515602879

32. Lundahl B, Moleni T, Burke BL, Butters R, Tollefson D, Butler C, et al. Motivational interviewing in medical care settings: a systematic review and meta-analysis of randomized controlled trials. Patient Educ Couns. 2013;93(2):157-68. https://doi.org/10.1016/j.pec.2013.07.012

33. Machado Ramos S, López Otero E, Torres Garri LI. Intervención educativa para elevar los conocimientos y modificar hábitos sobre salud bucal en embarazadas. Mediciego. 2012;18(1):1-7. Disponível em: http://bvs.sld.cu/revistas/mciego/vol18_01_2012/articulos/t-7.html

34. Marcenes W, Kassebaum NJ, Bernabe E, Flaxman A, Naghavi M, Lopez A, et al. Global burden of oral conditions in 1990-2010: a systematic analysis. J Dent Res. 2013;92(7):592-7. https://doi.org/10.1177/0022034513490168

35. Marinho VC, Higgins JP, Logan S, Sheiham A. Topical fluoride (toothpastes, mouthrinses, gels or varnishes) for preventing dental caries in children and adolescents. Cochrane Database Syst Rev. 2003(4):CD002782. https://doi.org/10.1002/14651858.CD002782

36. McHugo GJ, Drake RE, Whitley R, Bond GR, Campbell K, Rapp CA, et al. Fidelity outcomes in the National Implementing Evidence-Based Practices Project. Psychiatr Serv. 2007;58(10):1279-84. https://doi.org/10.1176/appi.ps.58.10.1279

37. Miller WR, Rose GS. Toward a theory of motivational interviewing. Am Psychol. 2009;64(6):527-37. https://doi.org/10.1037/a0016830

38. Minah G, Lin C, Coors S, Rambob I, Tinanoff N, Grossman LK. Evaluation of an early childhood caries prevention program at an urban pediatric clinic. Pediatr Dent. 2008;30(6):499-504.

39. Mohebbi SZ, Virtanen JI, Vahid-Golpayegani M, Vehkalahti MM. A cluster randomised trial of effectiveness of educational intervention in primary health care on early childhood caries. Caries Res. 2009;43(2):110-8. https://doi.org/10.1159/000209343

40. Moher D, Dulberg CS, Wells GA. Statistical power, sample size, and their reporting in randomized controlled trials. JAMA. 1994;272(2):122-4. https://doi.org/10.1001/jama.1994.03520020048013

41. Moher D, Liberati A, Tetzlaff J, Altman DG, Group P. Preferred reporting items for systematic reviews and meta-analyses: the PRISMA statement. PLoS Med. 2009;6(7). https://doi.org/10.1371/journal.pmed.1000097

42. Moskovitz M, Abud W, Ram D. The influence of an oral health education program provided in a community dental clinic on the prevalence of caries among 12-14 year-old children. J Clin Pediatr Dent. 2009;33(3):259-64. https://doi.org/10.17796/jcpd.33.3.r3p7351I5x52737u

43. Palacio A, Garay D, Langer B, Taylor J, Wood BA, Tamariz L. Motivational Interviewing Improves Medication Adherence: a Systematic Review and Meta-analysis. / Gen Intern Med. 2016;31(8):929-40. https://doi.org/10.1007/s11606-016-3685-3

44. Pereira MB, do Carmo Matias Freire M. An infant oral health programme in Goiania-GO, Brazil: results after 3 years of establishment. Braz Oral Res. 2004;18(1):12-7. https://doi.org/10.1590/S1806-83242004000100003

45. Plutzer K, Spencer AJ. Efficacy of an oral health promotion intervention in the prevention of early childhood caries. Community Dent Oral Epidemiol. 2008;36(4):335-46. https://doi.org/10.1111/j.1600-0528.2007.00414.x

46. Raj S, Goel S, Sharma VL, Goel NK. Short-term impact of oral hygiene training package to Anganwadi workers on improving oral hygiene of preschool children in North Indian City. BMC Oral Health. 2013;13(67):1472-6831. https://doi.org/10.1186/1472-6831-13-67

47. Ramos-Gomez FJ, Shepard DS. Cost-effectiveness model for prevention of early childhood caries. J Calif Dent Assoc. 1999;27(7):539-44.

48. Schulz KF, Grimes DA. Sample size calculations in randomised trials: mandatory and mystical. Lancet. 2005;365(9467):1348-53. https://doi.org/10.1016/S0140-6736(05)61034-3 
49. Sheiham A, James WP. A new understanding of the relationship between sugars, dental caries and fluoride use: implications for limits on sugars consumption. Public Health Nutr. 2014;17(10):2176-84. https://doi.or/10.1017/S136898001400113X

50. Shwartz M, Gröndahl HG, Pliskin JS, Boffa J. A longitudinal analysis from bite-wing radiographs of the rate of progression of approximal carious lesions through human dental enamel. Arch Oral Biol. 1984;29(7):529-36. https://doi.org/10.1016/0003-9969(84)90074-8

51. Si Y, Guo Y, Yuan C, Xu T, Zheng SG. Comprehensive Oral Health Care to Reduce the Incidence of Severe Early Childhood Caries (s-ECC) in Urban China. Chin J Dent Res. 2016;19(1):55-63. https://doi.org/10.3290/j.cjdr.a35698

52. Silva TFC. Assessing fidelity in psychosocial interventions: a systematic literature review. J Bras Psiquiatr. 2014;63(3):260-71. https://doi.org/10.1590/0047-2085000000034

53. Solomon ES, Voinea-Griffin AE. Texas First Dental Home: A Snapshot after Five Years. Tex Dent J. 2015;132(6):382-9.

54. Strippel $\mathrm{H}$. Effectiveness of structured comprehensive paediatric oral health education for parents of children less than two years of age in Germany. Community Dent Health. 2010;27(2):74-80. https://doi.org/10.1922/CDH_2223Srtippel07

55. Twetman S. Prevention of early childhood caries (ECC)--review of literature published 1998-2007. Eur Arch Paediatr Dent. 2008;9(1):12-8. https://doi.org/10.1007/BF03321590

56. US Preventive Services Task Force, Grossman D, Bibbins-Domingo K, Curry S, Barry M, Davidson KW ea. Behavioral Counseling to Promote a Healthful Diet and Physical Activity for Cardiovascular Disease Prevention in Adults Without Cardiovascular Risk Factors: US Preventive Services Task Force Recommendation Statement. JAMA. 2017;318(2):167-74. https://doi.org/10.1001/jama.2017.7171

57. Vachirarojpisan T, Shinada K, Kawaguchi Y. The process and outcome of a programme for preventing early childhood caries in Thailand. Community Dent Health. 2005;22(4):253-9.

58. Wagner Y, Greiner S, Heinrich-Weltzien R. Evaluation of an oral health promotion program at the time of birth on dental caries in 5-year-old children in Vorarlberg, Austria. Community Dent Oral Epidemiol. 2014;42(2):160-9. https://doi.org/10.1111/cdoe.12072

59. Watt RG. Strategies and approaches in oral disease prevention and health promotion. Bull World Health Organ. 2005;83(9):711-8. https://doi.org/10.1111/j.1600-0528.2007.00348.x

60. Watt RG. From victim blaming to upstream action: tackling the social determinants of oral health inequalities. Community Dent Oral Epidemiol. 2007;35(1):1-11. https://doi.org/S0042-96862005000900018

61. Whittle JG, Whitehead HF, Bishop CM. A randomised control trial of oral health education provided by a health visitor to parents of pre-school children. Community Dent Health. 2008;25(1):28-32. https://doi.org/10.1922/CDH_2143Whittle05

62. World Health Organization. Integrated health services - what and why. Geneva: WHO; 2008 [citado 30 nov 2017]. (Technical Brief, 1).Disponível em: http://www.who.int/healthsystems/ service_delivery_techbrief1.pdf

Contribuição dos Autores: Concepção e planejamento do estudo: AMM, AMC. Coleta, análise e interpretação dos dados: AMM, AMC, AERS. Elaboração ou revisão do manuscrito: AMM, AMC. Todos os autores aprovaram a versão final do manuscrito e assumem a responsabilidade pública pelo seu conteúdo.

Conflito de Interesses: Os autores declaram não haver conflito de interesses. 